\title{
ZnO nanoarray based conductometric acetone sensor: a bias and light assisted performance enhancement
}

\author{
Syed Sulthan Alaudeen Abdul Haroon Rashid ${ }^{1}$, Ylias M. Sabri ${ }^{1}$, Ahmad Esmaielzadeh Kandjani ${ }^{1}$, \\ Suresh K. Bhargava ${ }^{1}$, Antonio Tricoli ${ }^{2}$, Woitek Wlodarski ${ }^{3}$ and Samuel J. Ippolito ${ }^{3,{ }^{*}}$ \\ ${ }^{1}$ Centre for Advanced Materials and Industrial Chemistry (CAMIC), School of Applied \\ Sciences, RMIT University, GPO Box 2476, Melbourne, Victoria 3001, Australia. \\ ${ }^{2}$ Nanotechnology Research Laboratory, Research School of Engineering, \\ The Australian National University, Canberra 2601, Australia. \\ ${ }^{3}$ Sensor Technology Laboratory, School of Electrical and Computer Engineering, RMIT University, \\ Melbourne, Victoria 3001, Australia. \\ E-mail: samuel.ippolito@rmit.edu.au
}

\begin{abstract}
In this work, a combination of different bias potentials $(0.1-9 \mathrm{~V})$ along with $365 \mathrm{~nm}$ UV light is applied to enhance the sensing performance of a chemo-resistive device consisting of zinc oxide (ZnO) nanoarrays (NAs) as the sensitive layer for measuring acetone concentrations between 1.2 and $12.5 \mathrm{ppm}$. To the best of the authors' knowledge, the application of this double enhancement principle towards chemo-resistive acetone sensors is the first such report in the literature. It was found that an increment in applied bias increased the sensitivity of the device toward acetone. Furthermore, when the sensor was stimulated with a $365 \mathrm{~nm}$ UV light source in the presence of $12.5 \mathrm{ppm}$ acetone, the sensitivity was increased by 1.8 times when compared to operating in the dark. The significant sensitivity enhancements are effects of both light and bias, which can be highlighted by the calculated $1 \mathrm{ppb}$ limit of detection (LoD) of the sensor when excited by UV light at $350{ }^{\circ} \mathrm{C}$ with a $9 \mathrm{~V}$ bias. Additionally the adsorption time ( $\left.\mathrm{t}_{90-\mathrm{a}}\right)$ and desorption time $\left(\mathrm{t}_{90-\mathrm{d}}\right)$ of the sensor can be greatly improved with bias and light. The $t_{90-a}$ decreased to from $75 \mathrm{~s}$ to $9 \mathrm{~s}$ and the $t_{90-\mathrm{d}}$ from $93 \mathrm{~s}$ to $14 \mathrm{~s}$ under light for an operating bias of 0.1 and $9 \mathrm{~V}$, respectively.
\end{abstract}

Key words: Chemo-resisitve, $\mathrm{ZnO}$ nanoarrays, Acetone sensing, Bias, Light

\section{Introduction}

Metal oxide semiconductor (MOS) based chemo-resistive sensors are widely studied for acetone sensing due to their high reproducibility, easy fabrication procedures and simple operation. Amongst the metal oxides investigated, $\mathrm{ZnO}$ has shown much promise due to its unique optical and semiconducting properties ${ }^{1}$. ZnO NAs morphology is preferred because of its high surface area to volume ratio, single crystalline nature and uniform structures. In addition, $\mathrm{ZnO}$ is highly responsive to ultra violet light with the charge carrier generation phenomena at the surface. It is crucial yet challenging to attain differentiable sensitivity to identify the different gas concentrations. Therefore, sensors with high gas sensitivity and lower detection limits of $<1$ $\mathrm{ppm}$ are essential to facilitate applications like the diagnosis of diabetes using exhaled breath ${ }^{2}$. To overcome such issues, this paper investigated the effect of combining both bias and UV light stimulus on a chemo-resistive based acetone sensor to improve its overall performance.

\section{Experimental}

$\mathrm{ZnO}$ was synthesized using a previously reported synthesis method ${ }^{3}$, and morphological analysis was performed using scanning electron microscopy (SEM). The acetone vapor sensing event (acetone exposure) and recovery process (which involved flushing the chamber with dry air) were each set at 5 minutes while maintaining a total flow rate of $200 \mathrm{sccm}$. The acetone target concentrations were chosen between 1.2 to $12.5 \mathrm{ppm}$. The acetone sensing performance was tested under operating temperatures ranging from $45^{\circ} \mathrm{C}$ to $350^{\circ} \mathrm{C}$ with the different potential bias ranging from 0.1 to $9 \mathrm{~V} \mathrm{DC}$ in a custom built chamber with a quartz window to expose UV light of incident intensity of $2.024 \mathrm{~mW} \cdot \mathrm{cm}^{-2}$ for light assisted acetone sensing.

\section{Results and discussion}

The developed $\mathrm{ZnO}$ NAs (Fig. 1a-b) are observed to be uniform in size and vertically oriented with a high density. 

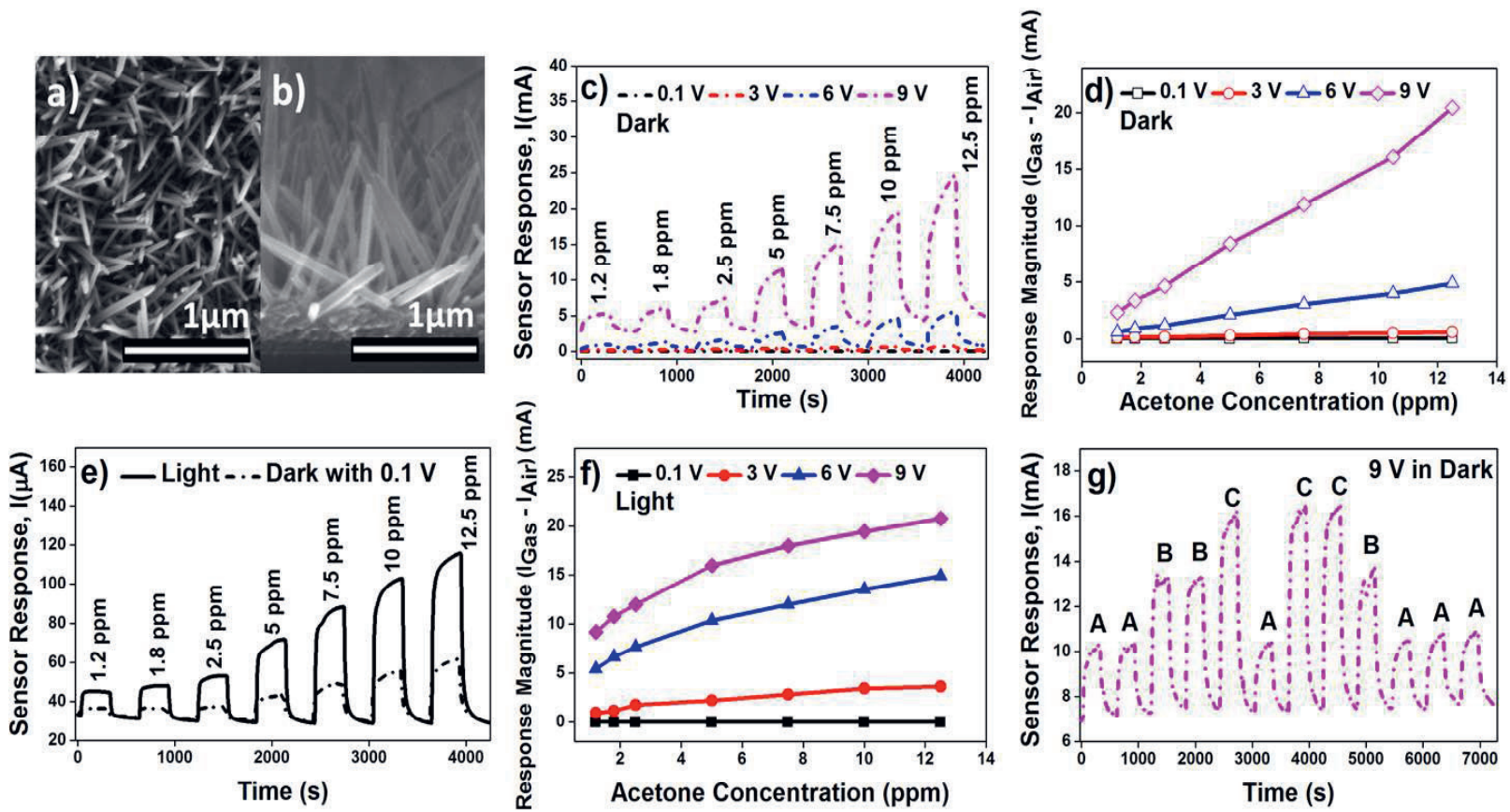

Fig. 1. SEM image: ZnO NAs (a) Top view (b) Side view; ZnO NAs sensor response towards acetone concentrations at an operating temperature of $350^{\circ} \mathrm{C}$ with different biases; (c) Dynamic response profile in the dark; (d) Calibration curve at different biases; (e) Dynamic response profile in dark and under UV light with 0.1 V bias; (f) Calibration curve under light with different bias; (g) Memory effect test in the dark using acetone concentrations of $A=1.2 \mathrm{ppm}, B=5 \mathrm{ppm}$ and $C=12.5 \mathrm{ppm}$ with $9 \mathrm{~V}$ bias.

The $\mathrm{ZnO}$ NAs have a length of approximately $1.2 \mu \mathrm{m}$ and are $75 \mathrm{~nm}$ in diameter. The sensitivity of the sensor toward acetone was observed to increase with increasing bias (see Fig. 1c). This is due to no charge mobility saturation of the semiconductor ${ }^{1}$. The sensitivity increased by 4.5 times more with $9 \mathrm{~V}$ bias than $6 \mathrm{~V}$ bias as shown in Fig. 1d. The ZnO NAs sensors were highly repeatable (calculated as repeatability $(\%)=100 \%-\operatorname{CoV}$ (Coefficient of Variance)) with $100 \%$ for $9 \mathrm{~V}$ bias and more than $97 \%$ for other bias values.

Table 1: LoD, adsorption time (t $\left.t_{90-a}\right)$ and desorption time (t $\left.t_{90-d)}\right)$ for UV assisted acetone sensor under different biases at $350^{\circ} \mathrm{C}$.

\begin{tabular}{|c|c|c|c|}
\hline $\begin{array}{c}\text { Bias } \\
(V)\end{array}$ & $\begin{array}{c}\text { LOD (ppm) } \\
(\text { Light) }\end{array}$ & $\begin{array}{c}\left(t_{90-\mathrm{a}}\right) \\
(\mathrm{s})\end{array}$ & $\begin{array}{c}\left(t_{90-\mathrm{d}}\right) \\
(\mathrm{s})\end{array}$ \\
\hline 0.1 & 0.07 & 75 & 93 \\
\hline 3 & 0.03 & 30 & 72 \\
\hline 6 & 0.006 & 29 & 34 \\
\hline 9 & 0.001 & 9 & 14 \\
\hline
\end{tabular}

Further sensitivity of the sensor under light towards acetone was observed to increase with the increasing bias as shown in Fig. 1f. The sensors exhibited almost no memory effect as shown in Fig. 19.
Interestingly, the combination of bias and light assisted sensing approach introduced in this study has enabled the sensor to operate at $45^{\circ} \mathrm{C}$ which reduced the efforts of doping or different material synthesis (data to be presented in the full manuscript). The influence of light and bias towards acetone with humidity as a cross-contaminant will also be presented in the final manuscript.

\section{Acknowledgments}

The support from the Australian Research Council through Discovery Project DP150101939 is gratefully acknowledged.

\section{References}

[1] Nasiri, N.; Bo, R.; Wang, F.; Fu, L.; Tricoli, A., Ultraporous Electron-Depleted ZnO Nanoparticle Networks for Highly Sensitive Portable Visible-Blind UV Photodetectors. Advanced Materials 2015, 27 (29), 4336-4343; 10.1002/adma.201501517

[2] Claire, T.; Christopher, W.; Shu, H.; Mark, E., Breath acetone concentration decreases with blood glucose concentration in type I diabetes mellitus patients during hypoglycaemic clamps. Journal of Breath Research 2009, 3 (4), 046004; 10.1088/1752-7155/3/4/046004 [3]Esmaielzadeh Kandjani, A.; Sabri, Y. M.; Mohammad-Taheri, M.; Bansal, V.; Bhargava, S. K., Detect, Remove and Reuse: A New Paradigm in Sensing and Removal of $\mathrm{Hg}$ (II) from Wastewater via SERS-Active $\mathrm{ZnO} / \mathrm{Ag}$ Nanoarrays. Environmental Science \& Technology 2015, 49 (3), 1578$1584 ; 10.1021 /$ es503527e 